
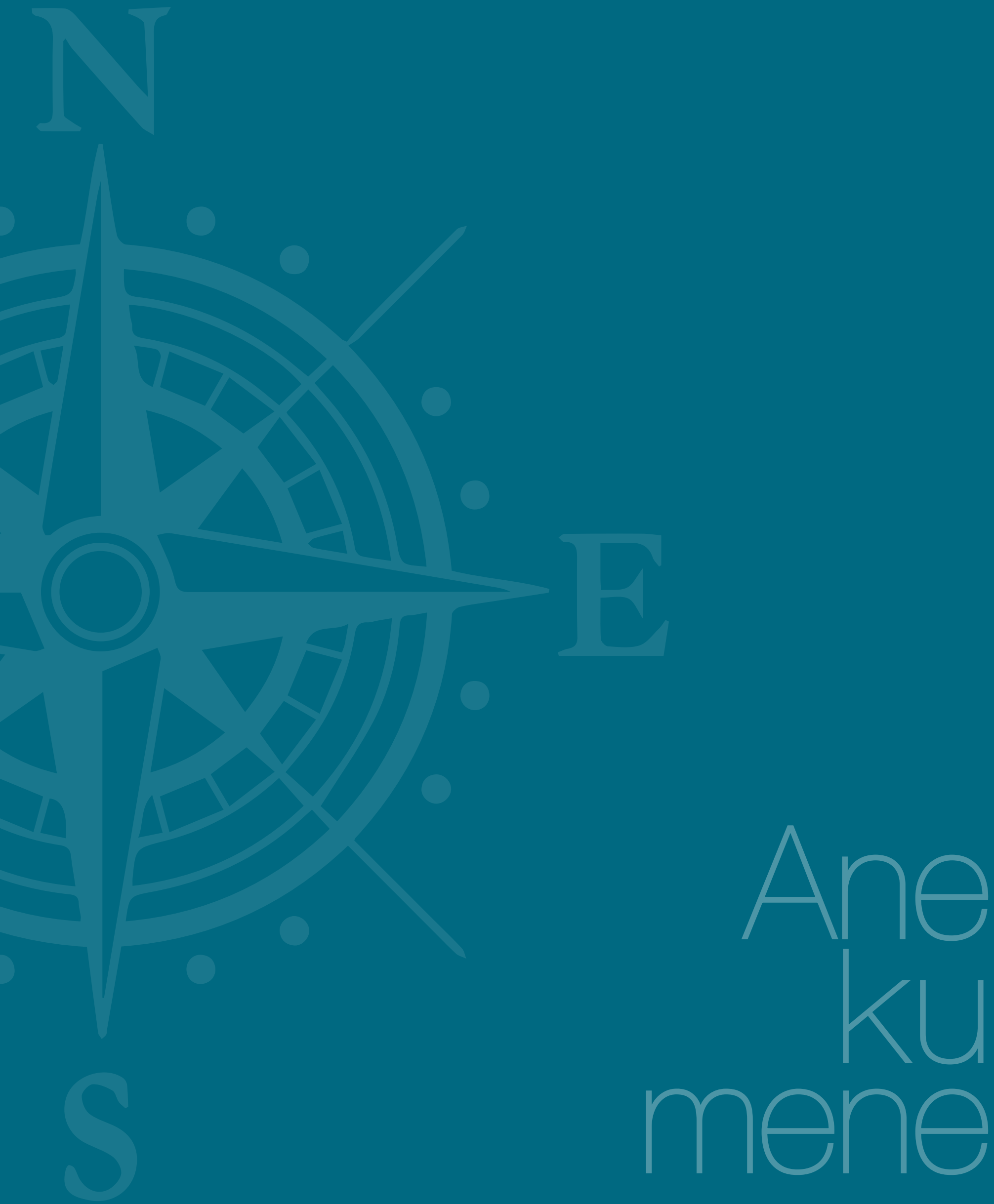


\title{
Mirada sobre un barrio en Bogotá
}

\section{A Glance at a Neighborhood from Bogotá Um olhar sobre um bairro em Bogotá}

\author{
María Camila Cely Moreno ${ }^{1}$
}

\section{Resumen}

Este artículo surge como ejercicio del Seminario de Énfasis en Vivienda de la Universidad Nacional, dirigido por el arquitecto Marco Cortés. Tiene como objetivo plantear una nueva mirada sobre el espacio cotidiano, el cual en el ámbito académico aún tiende a darse por sentado, y descubrir tanto problemas como soluciones urbanas en la ciudad del día a día de los estudiantes, para ir más allá de los ejemplos canónicos estudiados tradicionalmente en la escuela. Así, la mirada objetiva, condicionada por los conceptos aprendidos en clase, se mezcla con la actitud subjetiva de quien, efectivamente, habita el espacio, reconociendo elementos valiosos y, frecuentemente, invisibilizados. En este caso, se plantea dicha mirada ambigua sobre el barrio La Salle, en Bogotá. Los resultados del ejercicio fueron compartidos en clase, mostrando la diversidad de soluciones que pueden darse ante problemas comunes presentes en la ciudad.

\section{Palabras clave}

espacio cotidiano,

estructura urbana, vivienda

1 Arquitecta, Universidad Nacional de Colombia. 
Mirada sobre un barrio en Bogotá

María Camila Cely Moreno

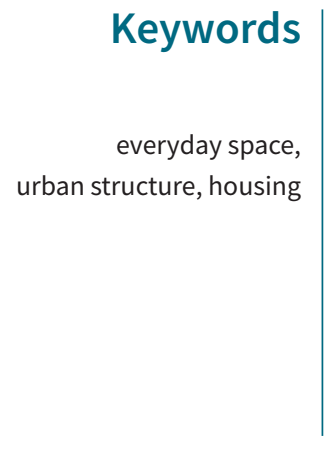

\section{Palavras-chave}

espaço cotidiano, estrutura urbana, habitação

\begin{abstract}
This article was written as an exercise at the Seminar of Housing Emphasis at Universidad Nacional de Colombia, leaded by the architect Marco Cortés. It pretends to give a new look at the everyday space, which is often neglected even in the academic field, and in this way, discover both problems and solutions for the students' everyday city, studying beyond the canonical examples traditionally studied at school. Thereby, the objective look determined by concepts learnt at class is combined with the subjective attitude of one who inhabits such space, recognizing valuable and often invisible elements. In this particular case, such ambiguous look is posed on La Salle neighborhood, in Bogotá. The results of these deliberations were shared in class, showing the diversity of solutions that may be developed given similar problems in the city.
\end{abstract}

\section{Resumo}

O presente artigo surgiu como um trabalho de aula no Seminário do Énfase em Habitação da Universidade Nacional, liderado pelo arquiteto Marco Cortés. O objetivo é dar uma nova olhada sobre o espaço cotidiano, que quase sempre se admite como dado mesmo no ambiente académico, e descubrir nele ambos problemas e soluções na cidade do dia a dia dos estudantes, indo além dos exemplos canónicos estudados tradicionalmente na escola. Nesse sentido, o olhar objetivo e determinado pelos conceitos aprendidos na aula mistura-se com a atitude subjetiva de quem realmente mora no espaço, permitindo reconhecer elementos valiosose freqüentemente invisíveis. Nesse caso, se olha ambiguamente o bairro La Salle, em Bogotá. Os resultados do trabalho foram compartilhados na aula, mostrando a diversidade de soluções que podem se presentar diante problemas comuns na cidade. 
El barrio La Salle se encuentra ubicado en la Localidad de Chapinero, un sector que originalmente albergaba casas de fin de semana de familias de élite, y que hoy día se ha convertido en una importante centralidad de Bogotá. Oficialmente, el barrio se encuentra delimitado al norte por la calle 62; al oriente, por la quebrada Las Delicias; al sur, por la Avenida Circunvalar y la calle 59, y al occidente, por la Avenida Carrera Séptima. Por su cercanía a los Cerros Orientales, el barrio se desarrolla en terreno inclinado, y la Carrera Séptima es su punto más bajo. La relación con esta vía jerárquica hace que sea un barrio con muy buena ubicación y fácil accesibilidad dentro de la urbe, pues se beneficia de las troncales de Transmilenio de la Avenida Caracas y la Carrera Séptima y de la amplia oferta de buses por la carrera 13 y la Carrera Séptima, así como de algunas ciclorrutas. Por hacer parte de un área que a diario congrega muchas actividades y a personas distintas, el barrio cuenta con fácil acceso a equipamientos institucionales, educativos, de salud, de ocio y de variedad de comercio. La ubicación del barrio se aprecia en el mapa 1.

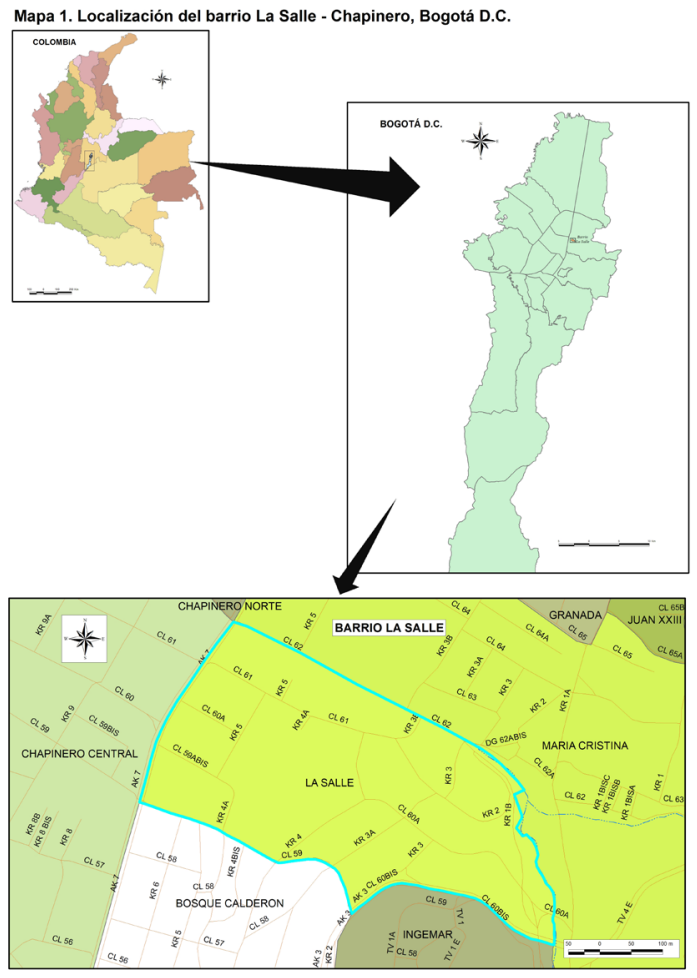

Figura 1. Barrio La Salle.

Fuente: elaboración propia.

Debido a esta concentración de comercio y servicios, la población itinerante del sector es muy alta y los flujos peatonales que se generan ocasionan la difusión de casi todos los límites del barrio. Esta difusión de límites se propicia porque las calles 59 y 62, e, incluso la Carrera Séptima no representan una barrera agresiva para el peatón, por lo cual fomentan la convivencia entre barrios aledaños.
Esta interacción constante entre barrios lleva a que no haya una legibilidad clara del barrio como una unidad independiente de su contexto, pues en la vida práctica se imbrica con los que lo rodean. Esta correlación se evidencia no solo morfológicamente, sino también en términos de actividad cotidiana, pues en un día cualquiera se puede, por ejemplo, sacar fotocopias en La Salle, ir al banco en el barrio María Cristina, hacer mercado en Chapinero Central y almorzar en Bosque Calderón; todo esto, recorriendo no más de cinco cuadras y sin que se sienta realmente que se ha pasado por cuatro barrios "distintos"; todos ellos son aspectos que se pueden apreciar en una aproximación a la representación cartográfica del barrio en el mapa 2.
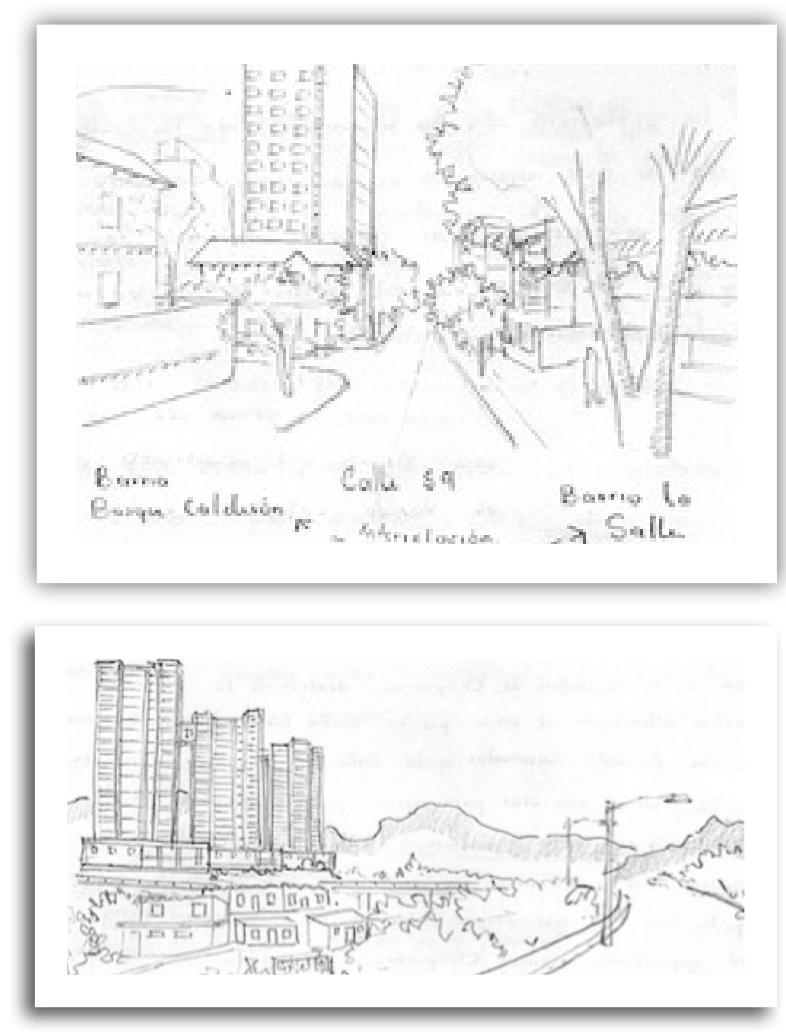

Figura 2. Espacios de contraste

Fuente: elaboración propia.

Sin embargo, contrastando con estos límites difusos (las calles perpendiculares a la Carrera Séptima), se encuentran los límites "superiores" del barrio: la quebrada Las Delicias y la Avenida Circunvalar. Estos actúan definitivamente como barreras infranqueables, debido a la fuerza de su escala, que no consigue articularse con el barrio. Adicionalmente, debido al descuido con el cual se han tratado las fuentes hídricas en la ciudad, en la quebrada Las Delicias existe un asentamiento informal de invasión. Los nuevos proyectos de vivienda que surgen en este sector expresan una clara voluntad de alejarse de dicha realidad, y crean con sus rejas de "seguridad" un ambiente hostil que hace que el borde sea aún más agresivo. 
Se podría pensar que la proximidad a vías de tan gran escala como la Avenida Circunvalary la Carrera Séptima impediría el adecuado desarrollo de la actividad residencial. Sin embargo, gracias a los criterios de implantación según los cuales el trazado vial se definió en función de las curvas de nivel, no existe en el barrio una calle que lo atraviese por completo y conecte de forma directa estas dos grandes vías. Así, el barrio queda aislado del tráfico denso y conserva la escala barrial de sus vías internas; además, privilegia los cambios de perspectiva que generan distintos niveles de privacidad y aprovechan a su favor las condicionantes del paisaje.

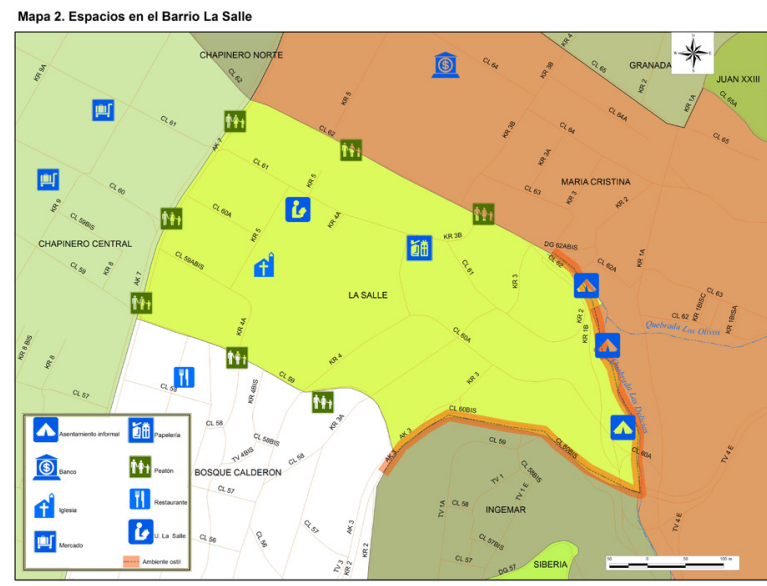

Fuente: elaboración propia.

Las vías se interrumpen debido no solo a la irregularidad del terreno, sino también, por la presencia de la Universidad de la Salle en medio del barrio. Además de darle nombre, la universidad le proporciona un aspecto del que de otro modo carecería: especificidad. Si bien se ha dicho que el barrio no se aísla de sus vecinos, la universidad permite, de cierta forma, su distinción del contexto; principalmente, debido a la Capilla de Nuestra Señora de la Estrella, elemento urbano legible y jerárquico por su posición y su forma.

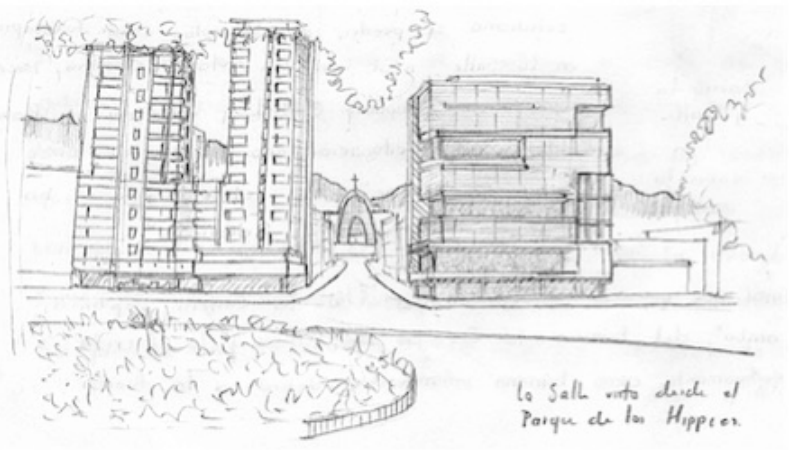

Figura 3. La Salle vista desde el Parque de los Hippies. Fuente: elaboración propia.
A pesar de ser esta iglesia una figura que contrasta con el fondo relativamente homogéneo y de llamar la atención, al ser visible desde diversos lugares del sector, su posible condición de centralidad del barrio se ve truncada por el muro perimetral, que la convierte en un edificio de acceso exclusivo para la universidad. Así, a pesar de su condición positiva de hito, tiene, a la vez, una condición desarticulada que desaprovecha su potencial de generar espacio público.

Sin embargo, esta desarticulación, queriéndolo o no, aporta elementos significativos para la configuración espacial del barrio, lo que evidencia la complejidad de su influencia. Al impedir el paso a través suyo, la universidad obliga, entonces, a rodearla. Esto, sumado a la irregularidad del terreno y del trazado vial, ayuda a mantener la privacidad de las calles interiores y enriquece el recorrido, al evitar su linealidad.

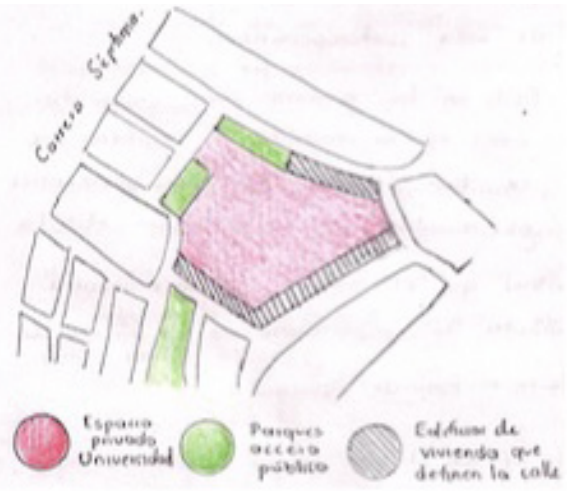

Figura 4. Caracterización de los usos del suelo en el barrio La Salle. Fuente: elaboración propia.

Un elemento importante: a pesar de su "enclaustramiento", entendido como una manera espacial de limitar o reducir una zona, la universidad prácticamente no le presenta fachadas cerradas al barrio. Así, resulta ser al mismo tiempo un elemento único que sirve de punto de referencia y un elemento homogéneo que se difumina con su contexto. La razón de esta dualidad está explicada en que el alma máter no es precisamente una manzana aislada y cerrada por todos sus costados, sino que tiene una fachada clara y todos sus otros costados están definidos por parques o por edificios de vivienda que asumen el papel de definir la calle.

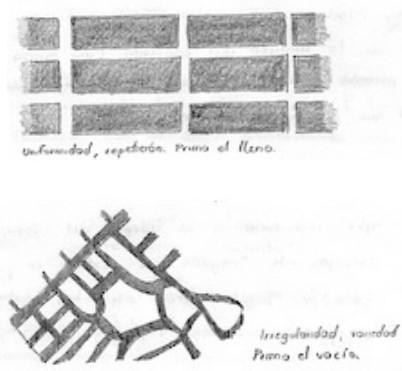


De este modo se hace evidente la ausencia del papel de representación de la manzana como unidad legible en el barrio: no puede entenderse la totalidad del barrio como una simple sumatoria de manzanas iguales. En este caso, la manzana no es una unidad, en primer lugar porque, como en el caso de la manzana de la universidad, existe una multiplicidad de usos, que aun cuando estén ubicados en el mismo "islote" no están relacionados entre sí; y en segundo lugar, porque al encontrarse definida por un trazado vial irregular tanto en planta como en alzado, no existe en el barrio una manzana igual a otra y la abstracción de una "manzana tipo" como unidad legible pierde fuerza en el imaginario de sus habitantes. En este sentido, el barrio no se entiende como un conjunto de manzanas conectadas por calles, sino como un conjunto de calles que configuran manzanas, donde cada una de dichas calles cuenta con una orientación, una perspectiva y un remate distintos, como se puede apreciar en el trazado de la representación anterior del espacio urbano.

De esta manera, la jerarquía de la calle en la composición del barrio se refuerza debido al desarrollo de vivienda por loteo y en manzanas compactas, contrario a los desarrollos cada vez mayores de vivienda en bloque suelto y aislado, donde la calle se convierte en un espacio residual, destinado, primordialmente, al paso de vehículos, y que se paramenta en la mayoría de los casos con rejas.

El desarrollo de vivienda por loteo es cada vez menos común, porque construir bloques repetitivos resulta más rentable y, en teoría, permite aprovechar la regularización de su diseño para generar áreas comunes dentro de la manzana, lo que no sucede cuando dicha manzana está dividida en múltiples edificios con distintas entradas. Sin embargo, tales áreas comunes resultan ser de uso exclusivo de los habitantes de dicha manzana en específico, que pasa a ser un "conjunto cerrado" de una única entrada, y que, en la práctica, es más nocivo para el sector, así tenga las publicitadas áreas comunes que un loteo tradicional.

Cuando varios edificios comparten la misma manzana se paramenta adecuadamente la calle, en vez de atacarla con rejas de "seguridad", y se propicia así una variedad urbana difícil de conseguir de manera planificada; variedad que, para perjuicio de las ciudades, se está dejando de lado por el afán de separarse de lo otro, de lo distinto, y por un ideal estético impuesto y obsesivo que busca lograr una uniformidad forzada.

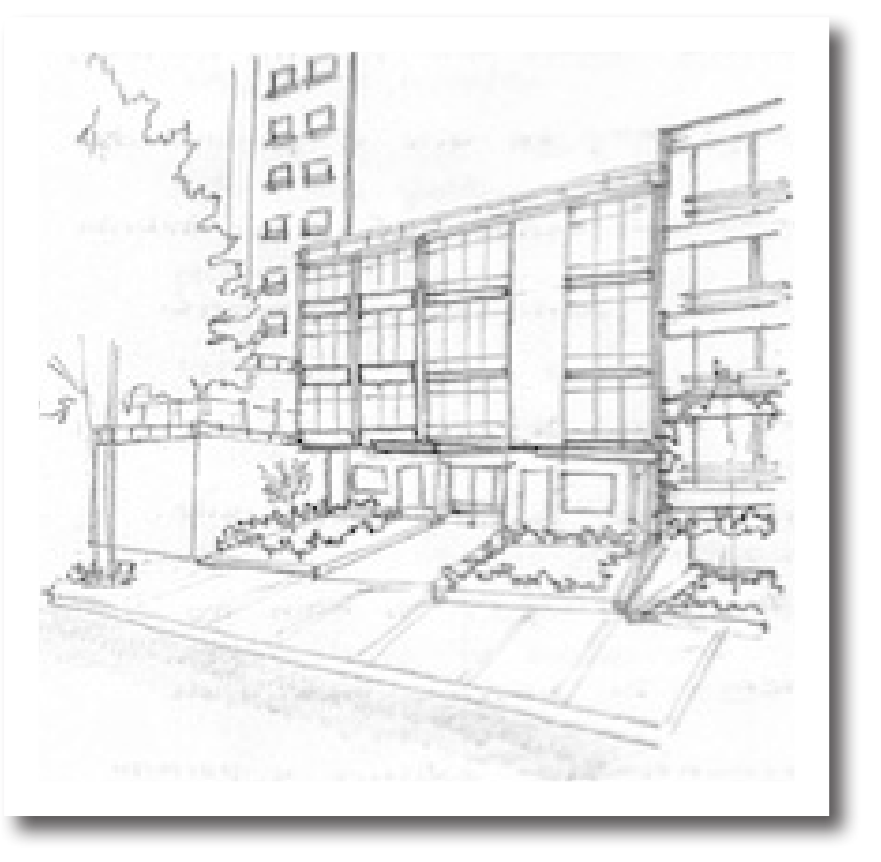

Figura 6. Vivienda en el barrio La Salle. Fuente: elaboración propia. 